\title{
Constructivismo, híbridos y pedagogía
}

\author{
José Esteban Pinto Ladino* \\ Victor Alonso Castro Bello ** \\ Orfa Mireya Siachoque Castillo *** \\ José Francisco Leguizamón Romero ${ }^{* * * *}$
}

Artículo de investigación

Fecha de Recepción: 19 mayo 2018.

Fecha de Aprobación: 10 septiembre 2018

\section{Resumen}

En la presente investigación se abordaron las tendencias pedagógicas de los docentes de la Institución educativa Magdalena, de la ciudad de Sogamoso (Boyacá); aspectos realizados en calidad de diagnóstico de un proyecto que pretendió apropiar en la praxis del área de matemáticas, el modelo constructivista social, como estrategia para mejorar el rendimiento académico de los estudiantes. Este proceso se analizó desde la clasificación propuesta por Porlán. La metodología propuesta para el estudio es cualitativa, con enfoque investigación-acción y de tipo descriptivo, puesto que integra dos recursos fundamentales como son la experimentación científica y la acción social. El análisis de los diferentes instrumentos aplicados para esta fase de la investigación, arroja como resultado que no hay una corriente pedagógica unificada, por lo tanto, se puede concluir que el profesorado tiene una tendencia híbrida, tomando de una y otra corriente lo que creen pertinente.

Palabras clave: constructivismo y constructivismo social, tendencias pedagógicas, currículo, práctica pedagógica, docente de secundaria, híbridos, educación.
* Institución Educativa

Magdalena-Sogamoso - Boyacá - Colombia jepint2@gmail.com

** Institución Educativa Magdalena-Sogamoso - Boyacá - Colombia vialcabe1972@gmail.com *** Institución Educativa

Magdalena-Sogamoso - Boyacá - Colombia yeyasiachoque@gmail.com **:***: Universidad Pedagógica y Tecnológica de Colombia Boyacá-Colombia francisco.leguizamón@uptc. edu.co

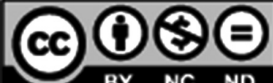




\section{Introducción}

Se contempla una tercera revolución educativa (Esteve, 2004), debido en primer lugar, al contexto macro que depende de la evolución de las fuerzas sociales (política y economía), en segundo lugar las leyes y decretos con capacidad de cambio limitada y por último la práctica docente, pues es el maestro quien finalmente tiene su propia mentalidad de enseñar. La educación contemporánea enfrenta muchos problemas, para transformarla se deben tener en cuenta factores fundamentales como la cultura profesional, las opiniones y las condiciones de trabajo de los profesores por su determinado contexto histórico.

En una primera instancia es el saber propio de los maestros, el que les permite orientar los procesos de formación de los educandos, el cual tiene como base histórica, propuestas significativas que los pedagogos han desarrollado a lo largo de los siglos. Dichas propuestas deben ser actualizadas y reconstruidas diariamente, pues cada clase es única y lo que hoy funciona con un grupo, mañana puede ser fallido, reflejándose estas acciones en los logros obtenidos.

Como educadores protagonistas inmersos en las dificultades de la educación actual, es del interés tomar alternativas de cambio en aspectos como la unificación y apropiación del modelo pedagógico institucional inmerso el currículo, que es la concreción de una teoría pedagógica por medio de la cual, se asegura el aprendizaje y el desarrollo de los estudiantes a la cultura de la época (Posner, 2005); luego en todo acto educativo institucional deben tenerse presente las perspectivas teóricas que favorecen a la comunidad educativa.

En la Institución Educativa Magdalena ubicada en el sur occidente de la ciudad de Sogamoso, de carácter oficial, se evidencian distintas tendencias en la praxis docente, no existe una inducción al modelo pedagógico y algunos docentes desconocen la tendencia pedagógica institucional. Este es un factor de gran peso en la parte coyuntural de la pedagogía , puesto que el modelo está determinado inicialmente por una teoría o paradigma que es adoptado por la institución en su Proyecto Educativo Institucional ( PEI) y el cual permite aplicar estas políticas en las aulas de clase.

En cuanto al currículo, está amparado por la ley general de educación, emanada de la constitución política de 1991 y plantea que éste debe ser flexible y con autonomía para cada institución educativa, da unos parámetros a seguir como los lineamientos curriculares y los derechos básicos de aprendizaje, los cuales son adoptados por las instituciones educativas como recetas que posibilitan buenos resultados en las pruebas externas, pero que son contraproducentes para la flexibilidad y autonomía docente (Romero, 2006).

Según el artículo 14 del decreto 1860 de 1994, "toda institución educativa debe elaborar y poner en práctica, con la participación de la comunidad, un proyecto educativo institucional que exprese la forma como se ha decidido 
alcanzar los fines de la educación definidos por la ley, teniendo en cuenta las condiciones sociales, económicas y culturales de su medio. El proyecto educativo institucional debe responder a situaciones y necesidades de los educandos, de la comunidad local, de la región y del país, ser concreto, factible y evaluable". Teniendo en cuenta lo anterior, para el presente proyecto se tomó como características del currículo las propuestas por Posner (2005), en donde se propone que el currículo no debe ser tomado como un plan de estudios, ni un esquema de contenidos por grados, sino que debe ser un objeto de acción simbólico y significativo, bajo enfoques epistemológicos y ciertos criterios de enseñanza que solo pueden comprenderse y ser comprobados en clase. Las características de este currículo se basan en cinco pasos: definir el concepto de hombre que queremos formar; caracterizar el proceso de formación del hombre; describir el tipo de experiencias educativas que se privilegian para afianzar e impulsar el proceso de desarrollo, incluyendo los contenidos curriculares; describir las regulaciones que permiten enmarcar $\mathrm{y}$ cualificar las interacciones entre el educando y el educador; describir métodos y técnicas utilizables en la práctica educativa como modelos de acción eficaces.

El currículo se ve afectado por las políticas nacionales, pues es el plan decenal de educación el que marca el derrotero en nuestro país, el cual se está gestando para el periodo 2016- 2026, concluyendo ya su primera fase de construcción; se trata de un material de
14 páginas en el que se definen los diez desafíos que tiene la educación para la próxima década. Uno de ellos es que en Colombia hay que dejar de enseñar lo mismo, como lo dice (Zubiría, 2017). "es necesario un profundo replanteamiento pedagógico de los currículos. Los modelos que enfatizan la transmisión de informaciones, hoy vigente en la mayoría de instituciones educativas, han demostrado que no logran promover el desarrollo humano e integral de los estudiantes. Los niños y los jóvenes presentan graves limitaciones para pensar, comunicarse y convivir, debido en parte, a la supremacía de los modelos pedagógicos tradicionales en la mayoría de colegios. Todos los docentes del país tendremos que aprender a partir de allí. El problema de la educación no es tecnológico sino pedagógico”.

Se debe entonces buscar un cambio en el currículo, lo cual significa cambiar los puntos de vista de los docentes acerca de los procesos de enseñanza y aprendizaje, y una intervención en su quehacer profesional, es escudriñar desde la más sencilla acción en clase siempre con el objetivo de mejorar día a día su práctica y lograr sus propósitos con cada estudiante, con cada grupo que se encuentre a su cargo (Olson, 1992).

Como educadores, protagonistas inmersos en las dificultades de la educación actual, es de interés, tomar alternativas para alcanzar una educación de calidad. Siguiendo la lógica clásica podría decirse, que si los docentes seguimos aplicando las "toda institución educativa debe elaborar y poner en práctica, con la participación de la comunidad, un proyecto educativo institucional que exprese la forma como se ha decidido alcanzar los fines de la educación definidos por la ley, teniendo en cuenta las condiciones sociales, económicas y culturales de su medio. El proyecto educativo institucional debe responder a situaciones y necesidades de los educandos, de la comunidad local, de la región y del país, ser concreto, factible y evaluable". 
mismas acciones, se obtendrán los mismos resultados, por lo que se hace necesario cambiar la praxis docente; según Bromme (1988), “es difícil cambiar la práctica si no se analizan las concepciones que están implícitas en ella y la naturaleza de los problemas concretos” (p 19), la educación actual se ve estereotipada por el curriculum tradicional, por cuanto se presenta una baja sensibilidad al cambio, debido a que se percibe el tradicionalismo como la manera normal posible de enseñar, transmitiendo contenidos de forma ergonómica, mediante marcador $\mathrm{y}$ saliva, en la que el conocimiento es la suma de unidades memorizadas, imposibilitando la formulación de hipótesis; lamentablemente hasta el momento estos son los instrumentos más empleados por los docentes al interactuar con los estudiantes (Porlán, 1995).

Para Elliott (2000), la intervención del profesor en el aula es un auténtico proceso de investigación, puesto que requiere diagnosticar los diferentes estados y movimientos de la compleja vida del aula, desde la perspectiva de quienes intervienen en ella, y elaborar, experimentar, evaluar y redefinir los modos de intervención en virtud de los principios educativos que justifican y validan la práctica y de la propia evolución individual y colectiva de los estudiantes.

La falta de fundamentos o finalidades claras frente al desarrollo de las prácticas pedagógicas, influencia negativamente el rendimiento escolar de los estudiantes, donde el docente juega un papel central, pues es quien, en discusión con los demás docentes, con los estudiantes y la comunidad educativa, selecciona y prioriza los componentes del currículo, para de este modo atender a los requerimientos del Proyecto Educativo Institucional (PEI) y a unas exigencias generales tanto de orden nacional como universal (MEN, 1994).

En la revista Pedagógica boyacense, número 19 (año 20), se afirma que se ha llegado a un punto de desequilibrio en el que predominan las comunicaciones y se presenta la posibilidad de replantear lo que ayer fue un éxito, rescatando los postulados básicos y las tesis preexistentes, como base para fundamentar el pensamiento y estilo del hombre actual y el sentido del docente contemporáneo.

Algunos postulados de base son propuestos por Porlán (1995), en su obra Constructivismo y Escuela, centrados en creencias implícitas que dominan la acción del docente y toma como referencia el currículo tradicional, el enfoque tecnológico, el enfoque espontaneista $\mathrm{y}$ finalmente busca una síntesis integradora, siendo esta última la manera responsable de encarar cambios verdaderos y no superficiales.

Dentro de los trabajos de investigación más cercanos a esta propuesta, que tomó como base la clasificación de tendencias pedagógicas contempladas por Porlán, es el titulado "Instrumento para identificar modelos pedagógicos en el Instituto Técnico Rafael Reyes de la ciudad de Duitama”. Esta 
investigación presentó un estudio cuyo objetivo principal fue identificar los modelos pedagógicos adoptados por los docentes de dicha Institución.

\section{Metodología}

El presente trabajo aborda el desarrollo de la primera fase del proyecto titulado "Apropiación Del Modelo Pedagógico Institucional En El Aula de Matemáticas”, que tiene como objetivo identificar en la praxis del docente su tendencia pedagógica; dando respuesta a las preguntas : ¿qué concepciones tienen los docentes de la IEM del modelo pedagógico institucional?, ¿Qué tendencias pedagógicas se evidencian en las prácticas de los docentes de la institución?; para los cual, se emplearon dos instrumentos de investigación, la encuesta estructurada, aplicada a treinta y dos docentes de la Institución, de las diferentes áreas tanto de educación básica como media y la entrevista semiestructurada, con el fin de contrastar la información obtenida e identificar las tendencias pedagógicas de los docentes de la institución.

Gráfico 1. Tomado de "Instrumento para identificar modelos pedagógicos en el Instituto Técnico Rafael Reyes de la ciudad de Duitama”.

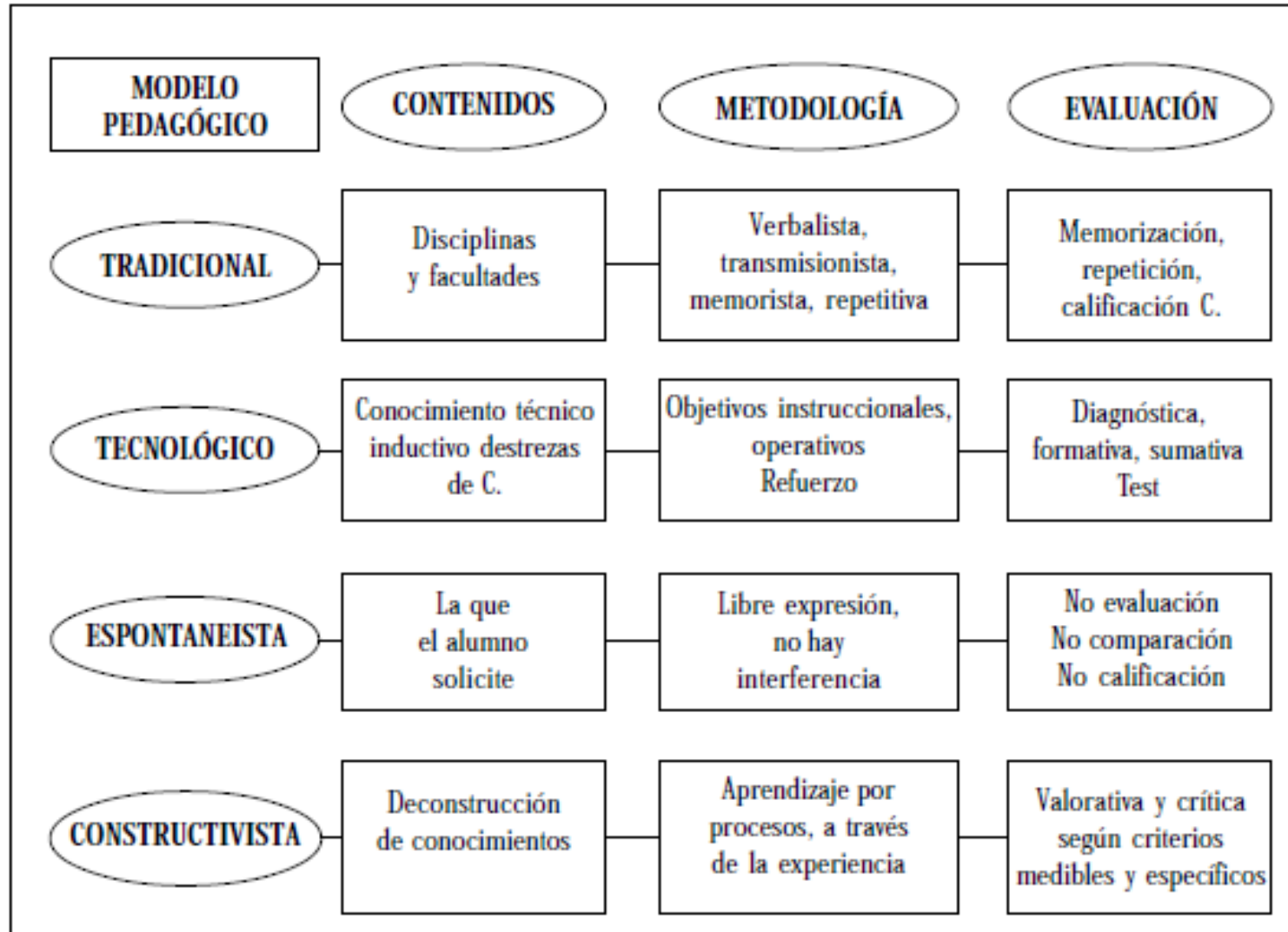


Como segundo instrumento de investigación se empleó una entrevista semiestructurada, aplicada a docentes de diferentes áreas de la institución, la cual consta de cinco preguntas, basadas en la teoría de Porlán, la cual pretendió identificar las concepciones de los docentes desde una mirada que apunta al modelo constructivista.

La metodología propuesta para el estudio es cualitativa, con enfoque investigación-acción y de tipo descriptivo. La investigación acción (IA) es un trabajo cíclico, en donde se conjugan la exploración, la actuación y la valoración de los resultados; es relevante para el presente trabajo, ya que se dedica al estudio de una situación social con miras al mejoramiento, en cuanto a la calidad de las diversas acciones que se llevan a cabo. También, porque se convierte en una forma de entender y comprender la enseñanza y no solamente, de investigar sobre ella, es una manera diferente de ver el oficio del maestro y de compartir su enseñanza, puesto que se realiza una permanente interacción humana, encaminada a una construcción colectiva de conocimientos.

\section{Resultados}

A continuación se presenta el análisis de la encuesta y de la entrevista semiestrucrada.

\section{Encuesta (Tendencias pedagógicas de la IEM).}

Una vez aplicado el primer instrumento de investigación (Tendencia pedagógicas presentes en los docentes de la IEM), se procede a realizar el análisis, inicialmente de acuerdo a la clasificación de las tendencias pedagógicas contempladas por Porlán, de donde se obtuvieron las siguientes reflexiones.

Tendencia tradicional. El docente de la IEM aun considera que el orden del salón de clase es fundamental para el buen desarrollo de la misma, partiendo de la ubicación de los estudiantes que deben estar dispuestos de tal manera, que solo observen al docente, donde el silencio y la disciplina son factores fundamentales para lograr un buen resultado académico. El docente se convierte en un prototipo dominante quien dice quien opina y quien no, evitando controversias que obstaculicen el desarrollo normal de la clase, siendo estos rasgos característicos del pensamiento pedagógico tradicional, como lo determina Porlán (1995, p 27) "Las conductas de aquellos alumnos que demuestran una falta de conexión con el hilo conductor de la clase, cuando no hay una clara interferencia con el esquema previsto (alumnos distraídos, contestaciones fuera de contexto, relaciones paralelas entre alumnos, etc.), son una expresión de la tendencia que poseen hacia la indisciplina y la Inadaptación escolar. Por el contrario, las conductas de aquellos alumnos que externamente demuestran atención y toman apuntes, son un reflejo fiel de que están siguiendo comprensivamente la explicación del profesor".

Tendencia tecnológica. De esta tendencia se puede interpretar que los docentes de la IEM tienen una fuerte 
influencia tecnológica, puesto que sus clases están orientadas al cumplimiento de objetivos, que son trazados por los derechos básicos de aprendizaje y/o los ejes temáticos de las áreas fundamentales, lo cual condiciona, en una buena parte las practicas docentes. De las diez preguntas planteadas en la encuesta, siete tuvieron aceptación.

Lo anterior permite inferir que los docentes están permeados en un alto grado por esta concepción pedagógica, donde se plantea la evaluación dirigida a la persecución de un logro ordenado de manera jerárquica, que fuerza a la uniformidad y favorece el aprendizaje mecánico e instrumental, preparando al estudiante para responder pruebas externas, sin valorar sus propias opiniones, que son las pautas de actuación características de la tendencia tecnológica según Porlán (1995, p. 32) "Qué enseñar? elaboración de una programación basada en objetivos operativos escalonados que conducen a objetivos de carácter terminal. Cómo enseñar? puesta en práctica de secuencias cerradas de actividades vinculadas a los objetivos operativos y escalonadas en el mismo sentido que ellos. Realización de un diagnóstico final, a través también de una prueba objetiva (test de opciones múltiples o similar), que mida el grado de consecución de cada objetivo terminal”. Entender el aprendizaje en una sola línea de acción irreversible, impide interpretar los procesos que manifiesta cada estudiante.

Tendencia espontaneista. De esta tendencia se evidencia que los docentes están medianamente permeados, teniendo en cuenta que aceptan cuatro de diez preguntas planteadas, de las cuales priman rasgos como la importancia de la motivación en el estudiante, sus intereses y privilegia el contexto cultural, social y económico, por encima de los contenidos, los objetivos y la evaluación.

El estudiante se educa en vinculación permanente con la realidad que lo rodea, desde la filosofía de que el aprendizaje verdadero se da como resultado de sus intereses y experiencia, y se halla en el entorno en que vive, se pretende despertar en el estudiante la observación y el descubrimiento, dejando de lado el conocimiento desde los contenidos supuestamente importantes en cada área. Desde esta tendencia, el docente prepara los contenidos teniendo en cuenta el escenario cultural en el que se evidencia el significado y la respuesta, que a su vez sirve de base para estimular la nueva pregunta, lo cual seguirá permitiendo la generación de otras nuevas preguntas que nacen del proceso mismo del estudiante.

Este proceso es una interacción activa entre el docente y el estudiante con una acomodación entreambos. Porlán(1995, p 41) plantea que "la enseñanza para que provoque realmente aprendizaje debe basarse fundamentalmente en las motivaciones espontáneas de los estudiantes. Modificación y readecuación permanente del plan de trabajo en función de los intereses y la motivación de los alumnos, utilizando estrategias de improvisación durante la misma acción”. 
Tendencia constructivista. Los docentes están de acuerdo en ocho de las diez preguntas que apuntan al constructivismo, es decir, consideran importante que el conocimiento sea un proceso individual, depende de los pre saberes, se aprende a partir de la problematización de lo que supuestamente ya se sabe, nunca el estudiante llega con una concepción vacía a cerca de un concepto, aprender es un proceso que no se realiza por transición directa, el profesor es un generador de ambientes de aprendizaje, la interacción entre estudiantes y estudiante-docente son básicos para lograr aprendizajes significativos, el reconocimiento de las necesidades es fundamental para lograr aprendizajes, se deben tener en cuenta los diferentes estilos cognitivos de los estudiantes.

Sin embargo, es de aclarar que la totalidad de los docentes de la institución no consideran importante la transformación conceptual del estudiante, es decir, que piensan que no se puede hacer una evolución del concepto del estudiante a pesar de todas las estrategias que pueda utilizar el docente. El proceso de evaluación que se lleva a cabo solo se dedica a verificar objetivos previamente planificados en el plan de área, evaluándose temas más no contenidos conceptuales

Bajo dos preguntas que pretendían identificar lo mismo (la importancia de la problematización del pre saber), se observa contradicción, es decir, resultados encontrados. La práctica pedagógica del docente se está desarrollando en torno a una guía sin tener en cuenta los pre saberes que tiene el estudiante de su contexto, ni la zona de desarrollo próximo, no se considera importante el enlace entre las estrategias para promover los conocimientos previos y la nueva información, lo cual potencia enlaces entre conocimientos previos, requeridos para lograr nuevos aprendizajes significativos (Díaz y Hernández, 1999).

Las zonas de desarrollo próximo son fundamentales en la construcción de un conocimiento constructivista como bien lo afirma Baquero (1996), evocando a Vygotski, el concepto de zona de desarrollo próximo (ZNP) se desarrolló a partir de las limitaciones y discusión del coeficiente intelectual (CI) entre distintas edades, buscando periodos para la adquisición de habilidades intelectuales y contextos referidos a dominios particulares. En términos generales son las acciones que el individuo al inicio puede realizar exitosamente (nivel actual real) y el desarrollo próximo potencial, para luego convertirse en un dialogo entre el niño y el futuro.

Para acceder a la zona potencial es importante el andamiaje, que es una interacción entre un sujeto de mayor experiencia y otro de menor experiencia para transformar al menos experto (Baquero, 1996).

A continuación se presenta el resultado global mediante una gráfica mostrando los resultados por tendencia.

En el análisis de esta primera herramienta se pudo evidenciar que el pensamiento pedagógico de los 


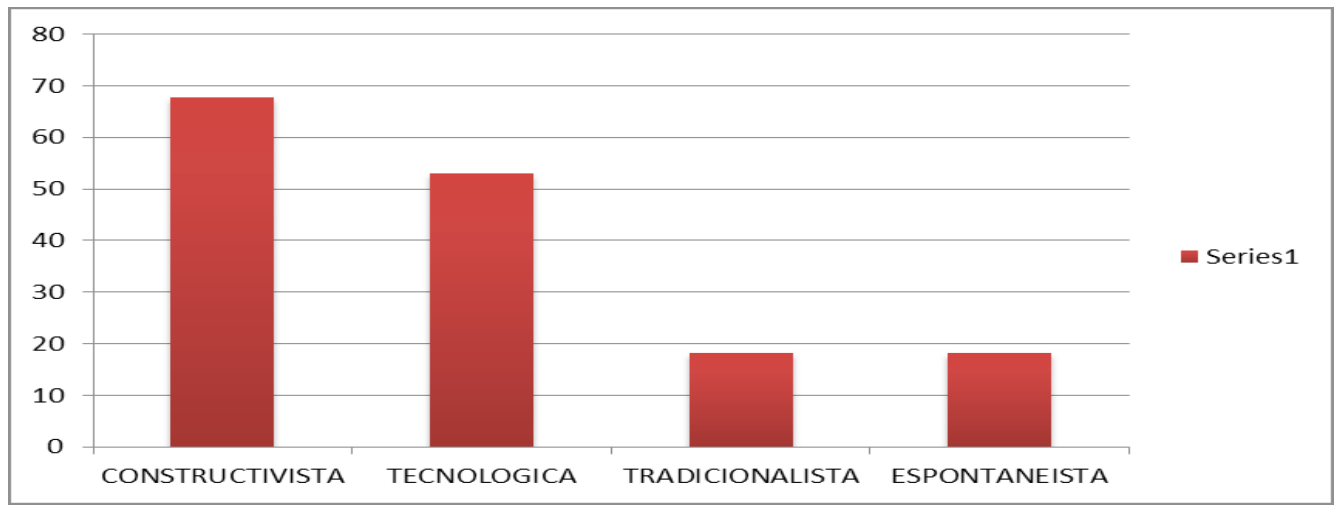

Gráfico 2. Resultados por tendencias.

Fuente: autor.

docentes de la institución, en su gran mayoría es constructivista, pero está influenciado por varias tenencias pedagógicas, primando la tendencia tecnológica desde la planeación de su clase, fijada en el cumplimiento de objetivos y metas que garanticen una enseñanza eficaz, observable $y$ cuantificable en el desempeño académico del estudiante; se evidenció que los docentes están permeados en menor medida y al mismo nivel por la tendencia espontaneísta y tradicional, por considerar un factor preponderante los intereses del estudiante, desarrollando el proceso de enseñanza desde su contexto socio cultural por encima de los contenidos, los objetivos y la evaluación, en la tendencia tradicional se evidenció que el profesor es considerado como un prototipo de autoridad, siendo un factor importante para conservar la disciplina, el orden y disposición de los estudiantes en el salón de clase.

\section{Entrevista semiestructurada.}

Se realizó la entrevista a los docentes de la institución, formulándoles cinco preguntas, teniendo en cuenta la tendencia constructivista desde la perspectiva de Porlán, contrastando las respuestas dadas por los docentes, obteniendo los siguientes resultados.

$¿$ Usted considera que los conocimientos previos que posee el estudiante sobre el tema son un obstáculo para el aprendizaje?

Todos los docentes coinciden en que los conocimientos previos del estudiante son importantes, porque se convierten en la base para adquirir un nuevo conocimiento o afianzar los conocimientos ya adquiridos. Un buen pre saber por parte del estudiante en la era de la información, hace que el docente esté en constante reflexión y actualización de su quehacer docente. 


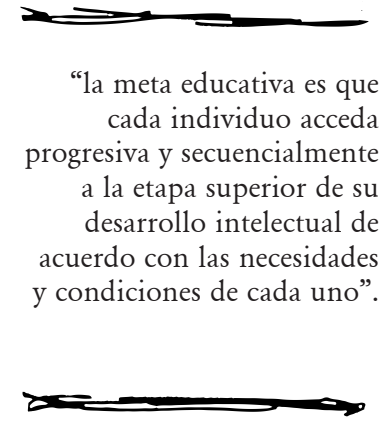

Los entrevistados coinciden en la importancia de los pre saberes y algunos van un poco más allá, notándose la tendencia constructivista, en donde el andamiaje lo comparan con una telaraña, como lo expresa el docente D5" No, yo creo que es al contrario, un buen aprendizaje debe basarse siempre en los conocimientos previos que son la base para que ellos enganchen los nuevos conocimientos que tienen, es como una especie de telaraña, haciendo la analogía, para que ellos de esa manera vayan construyendo su conocimiento sobre la base conceptual que ellos ya poseen, con base en las experiencias de vida que ellos ya han tenido".

La respuesta anterior guarda estrecha relación con lo expresado por Posner (2005, p 25), "la meta educativa es que cada individuo acceda progresiva y secuencialmente a la etapa superior de su desarrollo intelectual de acuerdo con las necesidades y condiciones de cada uno".

Porlán (1995, p105) afirma que"desde un punto de vista educativo, se trabaja desde y para el conocimiento que tienen, generan y construyen los alumnos", la postura de Porlán se evidencia en lo manifestado por el docente:

D22 "los obstáculos son importantes, son básicos para que el estudiante pueda entender los temas que se tratan en las clases, obviamente que él tiene que relacionar los conceptos previos con lo nuevo que ve en clase, para poder realmente tener un aprendizaje significativo".

¿El docente debe incentivar al estudiante para que utilice diferentes formas de representación de los problemas o situaciones pues le ayuda a aprenderlos?

Todos los docentes están de acuerdo con la importancia que tiene el hecho de que el estudiante tenga distintos puntos de vista frente a una situación determinada, en sus afirmaciones tienen en cuenta los diferentes métodos de aprendizaje de los estudiantes (inteligencias múltiples), una inteligencia implica la habilidad necesaria para resolver un problema o para elaborar productos que son importantes en un contexto cultural (Gardner, 1995). De las respuestas obtenidas por los docentes, la más afín con lo expresado por Gardner, fue manifestada por el docente:

D18 "A mí me parece que es importantísimo que los problemas se vean desde diferentes puntos de vista, si nosotros nos quedamos con una sola información acerca de un asunto, se va a cerrar la posibilidad de que el cerebro busque muchas soluciones, si lo vemos desde diferentes enfoques o desde diferentes temáticas, el mismo cerebro va a ayudar a que el estudiante aprenda que todos los problemas que se le presentan en la vida tienen diferentes soluciones y ayudan a que en su aprender académico tengan más responsabilidad y en sus problemas en la vida tengan amplitud para poderlos solucionar y así no acudan a las soluciones más rápidas”.

¿La enseñanza a partir de problemas de la vida cotidiana sirve especialmente para desarrollar en el estudiante el concepto? 
Todos los docentes coinciden en la importancia de la enseñanza a partir de problemas de la vida cotidiana, ya que esto motiva al estudiante en el aprendizaje, partiendo de una realidad conocida por él, lo cual permite una interiorización que genera una reestructuración interna del pre saber para modelar el nuevo concepto.

Esto pone en manifiesto que los docentes de la IEM, tienen una concepción mezclada entre rasgos tecnológicos y progresistas, ya que todos coinciden en afirmar que el aprendizaje se hace efectivo a partir de problemas de la vida cotidiana, como lo manifiesta D15 "Claro, es que es mejor aprender con algo que uno ha vivido desde la experiencia, es mejor aprender que dos manzanas más dos manzanas teniéndolas al frente, son cuatro y no simplemente dos números en el tablero, porque al saber un concepto a partir de la experiencia se graba en el corazón, en la mente y en el alma, entonces no solamente se tiene el concepto en la mente sino que se puede poner en práctica en todo, que es lo que debería ser la educación dar herramientas para la vida personal, académica, social”. Posner (2005, p 25) propone que "la meta educativa es que cada individuo acceda, progresiva y secuencialmente a la etapa superior de su desarrollo intelectual de acuerdo con la necesidades y condiciones de cada uno".

¿Los estudiantes aprenden fuera de la escuela aspectos sobre el tema, pero el aprendizaje real se da en la escuela?

A pesar de que todos los docentes no estén totalmente de acuerdo, si se evidencia la importancia que el docente le da al aprendizaje en el medio, teniendo en cuenta la teoría constructivista social (vygotskiana), que propone que el primer aprendizaje real comienza a darse de manera social en el medio que se desarrolla una persona $y$ en el momento en que comienza a aparecer el lenguaje.

El aprendizaje en el entorno es importante pero algunos docentes insisten, que debe necesariamente ser dirigido por la escuela, para obtener un buen aprendizaje, que lo convierta en un ser crítico y analítico, que sea capaz de interrelacionar su realidad con lo aprendido fuera y dentro de la escuela.

Apreciaciones importantes alrededor del aprendizaje son expresadas por el docente:

D4 "El aprendizaje, es un aprendizaje global, total, así que se aprende en la escuela, se aprende en la casa, se aprende en el barrio, se aprende en el parque, se aprende con los amigos, se aprende con la familia, se aprende en el colegio, porque eso no puede ser una cosa sesgada, el aprendizaje no solamente puede ser un cuaderno, un libro y un lápiz, el aprendizaje es la vida y si nosotros educaramos para la vida cambiaríamos radicalmente la sociedad".

La mente se considera como un espejo en el que reflejan objetos del mundo exterior (Font, 2000), es decir, la información que el estudiante adquiere del medio donde se desarrolla, se refleja en sus pensamientos y en su comportamiento. 
Es de notar que en el análisis cuantitativo realizado de la primera herramienta, como se observa en el gráfico 2 , muestra un fuerte porcentaje constructivista, correspondiente al $67.81 \%$
Dentro del marco legal, se contempla la educación como un proceso de formación permanente, personal $y$ social fundamentado en una concepción integral (Ley $\left.\mathrm{N}^{\circ} 115,1994\right)$.

¿Trabajar la temática de forma individual es la mejor estrategia que puede utilizar el docente, ya que si se permite que los estudiantes discutan o se comuniquen entre ellos se pierde el tiempo y no se obtienen rendimientos?

Se evidencia que para todos los docentes es relevante y de gran importancia el trabajo colaborativo, el cual ha sido aplicado por todos ellos, pero además este trabajo en algunos casos no ha sido tan exitoso, como lo manifiesta:

D5 "Yo pienso que el trabajo en equipo, no tanto en grupo, sino en equipo, es bien importante, siempre les recalco a ellos la importancia de la diferencia entre el trabajo en grupo y el trabajo en equipo, el trabajo en equipo es donde todos trabajan, todos aportan, diferente del trabajo en grupo que unos se recuestan en el trabajo de los otros y no tienen una participación activa; yo pienso que la escuela debe fomentar el trabajo en equipo, en esa forma, para que ellos hagan intercambio, discutan, debatan y sobre esa base ellos lleguen a acuerdos, a conclusiones que sean favorables para todo el equipo y para la sociedad también”.

El trabajo colaborativo, es un punto álgido en el aprendizaje constructivista, ya que es enriquecedor principalmente por el debate, el cual desarrolla en el estudiante la parte reflexiva y social
(Londoño, 2016), donde el estudiante conoce las ideas de sus compañeros, reflexiona sobre sus errores, para así entender que nadie tiene la verdad absoluta y que el proceso de aprendizaje es un proceso reflexivo y tolerante ante las diferencias de opinión. Porlán (1995) plantea que conseguir compaginar una participación espontánea con una dirección no autoritaria del proceso de aprendizaje debe ser una característica del docente constructivista.

\section{Conclusiones}

Es de notar que en el análisis cuantitativo realizado de la primera herramienta, como se observa en el gráfico 2, muestra un fuerte porcentaje constructivista, correspondiente al 67.81\%, siendo el mayor de los porcentajes obtenidos en la encuesta, al realizar un análisis cualitativo, se evidenció que en lo referente a la evaluación de la transformación del concepto, los docentes en su totalidad no dan importancia a este aspecto, siendo este uno de los principales fundamentos de la teoría constructivista.

De igual forma, en gran parte de las respuestas obtenidas en la entrevista, se evidenció la tendencia de los docentes a tener una concepción constructivista, ya que coinciden en aspectos como la importancia del aprendizaje a partir del pre saber de los estudiantes, el aprendizaje colaborativo, y la contextualización de éste, así como posibilitar a los estudiantes la posibilidad de plantear diferentes caminos para converger a la solución 
de una situación problémica a partir de los diferentes puntos vista.

En la institución educativa Magdalena, se encontró que pedagógicamente hablando el profesorado tiene una tendencia híbrida, esto indica que toman de una y otra corriente lo que creen pertinente para el desarrollo de sus clases, sin tener en cuenta los posibles efectos que esto acarrea en los procesos de enseñanza y aprendizaje, en su aspecto epistemológico, psicológico y ético. Lo anterior en su afán de cumplir y dar respuesta a las políticas educativas en su momento." No hay modelos puros, no hay pedagogías puras $y$ en ninguna institución encontraremos proyectos anclados en ellos, o hace falta mucho trabajo en las escuelas normales y en las facultades de educación para formar un maestro con un pensamiento pedagógico definido" (Nieto, 2008, p.204).

Cabe resaltar, que aún falta evidenciar si los pensamientos pedagógicos

manifestados en las herramientas aplicadas a los docentes, en esta primera fase, coinciden con la práctica en el aula, situación que nos ocupará en la segunda fase del proyecto.

\section{Referencias}

Baquero, R. (1996). Vigotsky y el aprendizaje escolar. Grupo editor S.A Madrid.

Bromme, R. (1988). Investigación y experiencias didácticas conocimientos profesionales de los profesores. Barcelona. Paidos.

Diario oficial No. 41.473 del 5 de Agosto de 1994.

Díaz, A., \& Hernández, G. (1999). Estrategias docentes para un aprendizaje significativo. Mexico. Editorial McGraw-Hill.

Elliot, J. (2000). La investigación-acción en educación. Editorial Morata.

Esteve J. (2004). La tercera revolución educativa: la educación en la sociedad del conocimiento. Barcelona Paidos Iberica.

Font, V. (2000). Consideraciones sobre la didáctica de las Matemáticas en la formación inicial del maestro. Educar.

Gardner, H. (1995). Inteligencias múltiples. La teoría en la práctica. Barcelona. Editorial Paidos.

Londoño, C. (2016). Constructivismo y teoría de la historia. Cuestiones De Filosofía, (16), 161-184. https://doi.org/10.19053/01235095.3954 
MEN. (1994). Decreto 1860 de Agosto 3 de 1994.

Nieto, L. (2008). Instrumento para identificar modelos pedagógicos en el Instituto Técnico Rafael Reyes de la ciudad de Duitama.

Ley N 115. Ley General de Educación de la República de Colombia, Bogota,1994.

Olson J. (1982). Innovation in the science curriculum. London. Croom Helm.

Porlán, R. (1995). Constructivismo y escuela. Sevilla. Diada Editora. Pp 24-33.

Posner, G. (2005). Análisis Del Currículo. p 24. Editorial McGraw-Hill, Tercera edición. Revista Pedagógico boyacense (19).

Romero, F. (2006). Análisis de necesidades de las instituciones educativas. Diálogos Educativos. Año 6, No. 11. ISSN 0718-1310. 